\title{
Entspannung durch das Hören einer Klangschale: Bevorzugte Klangmerkmale
}

\author{
Wolfgang Thies
}

\section{Einleitung}

„Klangschalen haben eine ganz besondere Qualität, die sich in dieser Art und Intensität nur bei ihnen einstellt: Ihr Klang wirkt auf die Mehrzahl aller Menschen tief entspannend“ (Lindner u. Mempel 2008, S. 20). Diese Aussage - gegründet auf reiche Erfahrungen mit der Anwendung von Klangschalen - lässt sich gut nachvollziehen, wenn man die zahlreichen anderen aus der Praxis hervorgegangenen Veröffentlichungen über Klangschalen liest: Unter den positiven Wirkungen, die sich den Berichten zufolge beim Hören von Schalenklängen einstellen können, steht die Entspannung (bzw. Beruhigung) an erster Stelle. Was Praktiker hierzu aus ihrem Erfahrungsschatz mitteilen, wurde allerdings wissenschaftlich nur ansatzweise untersucht. In wissenschaftlichen Studien zur Wirkung von Klangschalen auf den Menschen sind die von den Schalen ausgehenden Schallwellen meist mit weiteren Stimuli verknüpft, beispielsweise Schall aus zusätzlichen Quellen oder Körperkontakt der Schalen. Der Körperkontakt schwingender Klangschalen verknüpft den auditiven Reiz mit einem vibrotaktilen Reiz.

Ein Überblick zur Literatur über Klangschalen ist enthalten in Thies (2010), S. 38-39. Inzwischen erschienen weitere praxisorientierte Bücher (Draxler-Zenz 2016, Dülmen 2013, Hess 2015, Hess 2016, Lahner 2012, Lahner 2013, Lahner 2015, Leon 2012, Lindner 2014, Mayer 2013, Mempel 2012, Oberle 2013, Perry 2014, Pöttinger 2014, Wulf 2014, Wulf 2016). Darüber hinaus wurden einige wissenschaftliche Aufsätze veröffentlicht: Bidin et al. (2016) führten eine medizinische Pilotstudie mit zwölf Patienten durch. Klangschalen auf dem Körper der Patienten wurden sanft angeschlagen, ohne dabei $\mathrm{zu}$ sprechen. In geringem Umfang traten weitere Schlaginstrumente hinzu. Physiologische Parameter zeigten signifikant eine entspannende Wirkung. Braun u. Braun (2015) untersuchten an 100 Patienten mit Fibromyalgie-Syndrom die Wirkung von Klangschalen als zusätzliche Komponente einer stationären multimodalen Schmerztherapie. Die Schalen wurden „im Rahmen 
einer Fantasiereise" angeschlagen (S. 197). In der Gruppe mit Klangschalenanwendung war die Schmerzreduktion signifikant stärker ausgeprägt. Eine Untersuchung von Landry (2014) mit 51 Teilnehmern ergab, dass eine geführte Meditation signifikant besser entspannte, wenn die Probanden vor der Meditation eine live gespielte Klangschale anhörten. (Ausführlicher beschreibt die Autorin ihre Forschung in Landry 2012 und in Landry 2015.) Stamou et al. (2016) prüften in einer Pilotstudie mit 24 Drogenabhängigen, ob sich der Behandlungserfolg durch Einbeziehung von Musik verbessern lässt. Das „standard treatment“ der Kontrollgruppe wurde in einer Gruppe ergänzt durch ,systematic desensitization [...] combined with listening to slow-paced low-pitched meditation music without lyrics improvised live on Tibetan bowls" (S. 38). Die Verfasser stellten signifikante positive Effekte gegenüber der Kontrollgruppe fest. „The results of our study indicate that [...] listening to relaxing music combined with systematic desensitization may be useful [...] by reducing craving responses to drugconditioned cues and facilitating the enhancement of coping skills for confronting highrisk situations“ (S. 43). Die Autoren räumen ein: „Further research is [...] necessary in order to distinguish the effects of the music [...] from those of S[ystematic]D[esensitization]“ (S. 43). Aarts, Ouweltjes u. Bulut (2014) sowie Pawłowski, Portalska u. Portalski (2013) befassen sich mit der Akustik von Klangschalen und mit Möglichkeiten, Schalenschall und Varianten elektronisch zu synthetisieren. Aarts et al. haben ein elektroakustisches Gerät entwickelt, die „eBowl“. Sie hat die Größe einer mittelgroßen Klangschale und soll mit drei Lautsprechern in der Oberseite ähnlich räumlich klingen. Die elektrodynamisch in Schwingungen versetzte Unterseite überträgt wie eine Klangschale Vibrationen auf den menschlichen Körper. In einem Versuch mit zwölf Personen gab die eBowl auf dem Bauch der liegenden Teilnehmer Aufnahmen einer realen Klangschale wieder. Die Mehrzahl der erhobenen physiologischen Parameter zeigte eine signifikante Förderung der Entspannung durch die eBowl. Die akustische Studie von Collin, Keefer u. Moore (2015) ist dem Reiben des Schalenrands mit dem Schlägel gewidmet. Meyer u. Portalski (2013) sowie Smutný u. Pazdera (2014) behandeln die Akustik von Klangschalen im Zusammenhang mit der Messtechnik. Terwagne u. Bush (2011) stellen die akustischen Grundlagen dar und konzentrieren sich dann auf die Hydrodynamik einer flüssigkeitsgefüllten Klangschale.

Eine eigene Untersuchung (Thies 2008) diente dem Ziel, die alleinige Wirkung des Schalenklangs zu erkunden. 60 Personen hörten in Einzelsitzungen sieben Minuten lang eine live angeschlagene mittelgroße Klangschale. Während der anschließenden mündlichen Befragung nannten die Versuchsteilnehmer Entspannung als häufigste 
Wirkung. $80 \%$ der Beteiligten sagten, die Klänge hätten sie beim Entspannen unterstützt; $92 \%$ unter ihnen sprachen Entspannung bzw. Beruhigung an, bevor der Versuchsleiter das Thema erwähnte. Wichtig für die Entspannung waren nach Ansicht der Teilnehmer das Fluktuieren des Klangs und das allmähliche Verklingen. Hier setzt die im Folgenden beschriebene Studie an: Ein Hörversuch mit wirklichkeitsnah synthetisierten Schalenschwingungen soll den Zusammenhang dieser Klangeigenschaften mit der entspannenden Wirkung ausloten.

\section{Konzeption dieser Studie}

Der oben erwähnte Hörversuch (Thies 2008) hat gezeigt, dass das Hören einer Klangschale die Entspannung fördern kann. Vier Fünftel der Teilnehmer bezeichneten die Schalenklänge ohne Einschränkung als angenehm; ebenso viele gaben an, die Klänge hätten sie beim Entspannen unterstützt. Bei den Antworten auf die Fragen „Was war für Sie an den Klängen angenehm?“ und „Wodurch haben die Klänge Ihre Entspannung unterstützt?“" standen das Fluktuieren des Klangs und das allmähliche Verklingen im Vordergrund.

Die Geschwindigkeit der Klangfluktuation kann von Schale zu Schale sehr verschieden sein (Thies 2011). Das Verklingen dauert bei großen Schalen länger als bei kleinen; hier kommt als weiterer Klangunterschied die Tonhöhe ins Spiel. Ein weiterer Hörversuch soll Hinweise darauf geben, wie diese Klangeigenschaften mit der entspannenden Wirkung zusammenhängen. Dafür sind Audiosignale nützlich, deren Eigenschaften sich in genau bestimmter Weise ändern bzw. konstant halten lassen. Dies erreicht man am ehesten mit synthetisierten Schwingungen.

Ein Hörversuch mit vorgefertigten Aufnahmen anstelle einer live gespielten Klangschale ist nicht ideal: Beim genannten Hörversuch sagten $93 \%$ der Teilnehmer, sie würden die Schale lieber live hören als über Lautsprecher oder Kopfhörer. Der Verzicht auf die unmittelbare Wirkung einer realen Klangschale ist der Preis für die Möglichkeit, Klangeigenschaften gezielt und flexibel zu variieren. Auf die Frage nach der zweitbesten Art des Hörens - über Lautsprecher oder über Kopfhörer - zeigten die Teilnehmer des Hörversuchs keine klare Präferenz. Für den neuen Hörversuch dient ein geschlossener Kopfhörer, der unerwünschten Schall von außen dämpft. Weitere Vorteile gegenüber Lautsprechern sind die leichtere Reproduzierbarkeit der Versuchsbedingungen und die einfachere Handhabung beim Durchführen der Versuchsdurchläufe mit den einzelnen Teilnehmern in verschiedenen Räumen. Damit 
das Tragen des Kopfhörers nicht lästig wird, kann man ihn während des Versuchs mehrmals ablegen; außerdem ist der Versuchsablauf kurz gehalten. Die Audiosignale sind realitätsnah stereofon synthetisiert, sie klingen auch bei Kopfhörerwiedergabe recht räumlich.

Um den Einfluss bestimmter Schalleigenschaften zu untersuchen, hören die Versuchsteilnehmer Audiosignale, die sie paarweise vergleichen. Die Audiosignale unterscheiden sich in einigen Paaren nur durch die Geschwindigkeit des Fluktuierens; in anderen Paaren sind verschieden große Klangschalen simuliert, die (bei ähnlichem Fluktuieren) in verschiedener Tonlage unterschiedlich lang ausklingen. Der Hörversuch soll Aufschluss darüber geben, ob Menschen, die sich mit Hilfe von Schalenklängen entspannen möchten, eine Vorliebe für jeweils einen der paarweise gehörten Klänge zeigen. Der Einsatz eines Computers ermöglicht flexibles Anhören: Durch Mausklicks kann man die Klänge jedes Paars beliebig oft hören und jederzeit - ohne das Verklingen abzuwarten - einen Klang erneut von vorn abspielen oder zum anderen Klang wechseln. Jedem Paarvergleich folgt die Frage: „Nehmen wir an, Sie möchten sich entspannen. Meinen Sie, dass einer der Klänge (oder beide) Sie dabei unterstützen könnte?“ Bei positiver Antwort schließt sich die Frage an: „Welchen Klang würden Sie zum Entspannen lieber hören?“

\section{Die Audiosignale für den Hörversuch}

\subsection{Grundsätzliches}

Die Audiosignale für den Hörversuch werden synthetisiert. Die Synthese erlaubt es, die Geschwindigkeit des Fluktuierens gezielt zu ändern, ohne andere Merkmale zu beeinflussen. Außerdem ist es möglich, Schwingungen verschieden großer Klangschalen nachzubilden, die mit verschiedenen Grundfrequenzen länger oder kürzer ausschwingen, sich im Fluktuieren aber nur wenig unterscheiden. Wichtig ist ein wirklichkeitsnaher, auch bei Kopfhörerwiedergabe ausgeprägt räumlicher Klang, um dem Hören realer Klangschalen nahezukommen.

Das Fluktuieren des Klangs gehört zu den charakteristischen Merkmalen von Klangschalen. Es entsteht durch Aufspaltung der Teilschwingungen in zwei eng benachbarte Komponenten. Die dabei auftretenden Schwebungen führen zu ruhiger bis lebhafter Bewegung jedes Teiltons oder - bei hoher Schwebungsfrequenz - zu Rauigkeit. Obendrein kann bei tief liegenden Teiltönen eine räumliche Klangbewegung auftreten. Üblicherweise haben die einzelnen paarweise aufgespaltenen 
Teilschwingungen verschiedene Schwebungsfrequenzen; je nach den vorkommenden Schwebungsfrequenzen sind viele Bewegungsmuster möglich (Messergebnisse für 50 mittelgroße Klangschalen sind zusammengestellt in Thies 2011).

Klangschalen mit deutlich verschiedener Größe unterscheiden sich nicht nur durch die Grundfrequenz. Die Schwingung einer kleineren Schale ist stärker gedämpft (der Klang verebbt schneller), und die Amplitude der Teilschwingungen fällt zu den hohen Frequenzen hin stärker ab (man hört bei gleichartigem Anschlag weniger Teiltöne als bei einer größeren Schale). Die räumliche Klangbewegung ist bei kleineren Schalen weniger ausgeprägt.

Um die genannten akustischen Eigenschaften für den Hörversuch möglichst genau und unabhängig voneinander einstellen $\mathrm{zu}$ können, dient zur Herstellung der Audiosignale die sehr flexible additive Synthese: In beiden Stereokanälen sind für jede (aufgespaltene) Teilschwingung zwei Sinusschwingungen zusammengefügt. Hinzu kommt die Schwingung des Schlägels, die beim Anprall an die Klangschale entsteht. Sie wurde aus der Aufnahme eines Schalenanschlags isoliert und sorgt beim Anhören des fertigen Audiosignals für ein realistisches Anschlaggeräusch. Das Syntheseverfahren ist in einer früheren Veröffentlichung ausführlich beschrieben (Thies 2010, S. 44-48); dort sind auch die akustischen Eigenschaften von Klangschalen näher erläutert (S. 41-44).

\subsection{Variation der Schwebungsfrequenz}

Der Ausgangspunkt für die Synthese von Schalenschwingungen mit verschiedenen Schwebungsfrequenzen ist eine Aufnahme der in einem früheren Hörversuch live gespielten, mittelgroßen, dickwandigen Schale (links in Abb. 4), deren Klang viele Teilnehmer als angenehm und unterstützend beim Entspannen bezeichneten (Thies 2008). Die beiden Mikrofone waren ungefähr dort angeordnet, wo sich während des Hörversuchs die Ohren der Versuchsperson befanden: schräg oberhalb der Schale in einer Entfernung von etwa $70 \mathrm{~cm}$. Die Schale wurde bei der Aufnahme ähnlich wie im Hörversuch angeschlagen: nahe am Rand, radial von außen, mit einem dünn gepolsterten Holzschlägel. Die Schwebung aller Teilschwingungen ist gut hörbar, keine Schwebung ist auffallend stark ausgeprägt. Die Synthese umfasst die bei mittelstarkem Anschlag hörbaren fünf Teilschwingungen mit den Schwebungsfrequenzen 2,0 Hz, 1,9 Hz, 7,2 Hz, 5,7 Hz und 8,9 Hz (Klangbeispiel 1, Abb. 1). (Beim Anhören der Beispiele sollte die Lautstärke dezent eingestellt sein, damit der Klang realistisch wirkt. 
Die Versuchsteilnehmer konnten bis zum völligen Verklingen zuhören - bei der mittleren Schalengröße dauert dies etwa eine Minute - oder nach einer frei gewählten Zeitspanne abbrechen.)

Klangbeispiel 1: Mittelgroße Schale (Klang_1.mp3)

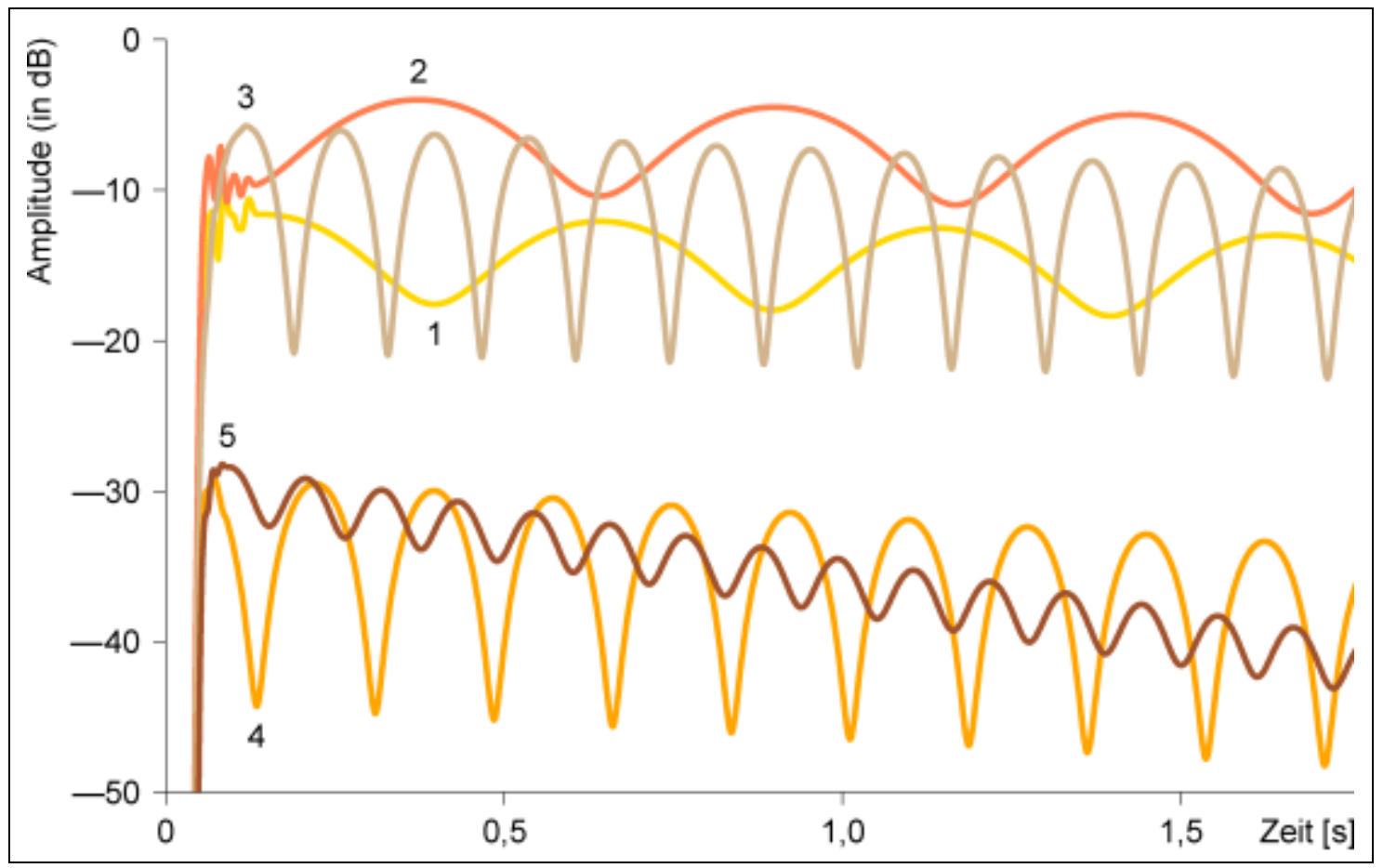

Abb. 1: Zu Klangbeispiel 1, Pegelverläufe der synthetisierten Teilschwingungen (linker Kanal)

Um die Schwebungsfrequenz einer Teilschwingung zu erhöhen bzw. zu verringern, vergrößert bzw. verkleinert man die Frequenzdifferenz der beiden Komponenten, in die jede Teilschwingung aufgespalten ist. Den Mittelwert der Frequenzen behält man bei - ebenso wie alle anderen Daten. Die auf diese Weise erhaltene Variante unterscheidet sich nur durch die Schwebungsfrequenz der bearbeiteten Teilschwingung vom ursprünglichen Audiosignal. Für den Hörversuch wurden mehrere Varianten hergestellt. Am lautesten klingt der zweite Teilton, hier sollten Änderungen also besonders gut hörbar sein. In der Variante ist die Schwebungsfrequenz der zweiten Teilschwingung verdoppelt (Klangbeispiel 2, Abb. 2). 
Klangbeispiel 2: Wie Klangbeispiel 1, aber Schwebungsfrequenz der zweiten Teilschwingung verdoppelt (Klang_2.mp3)

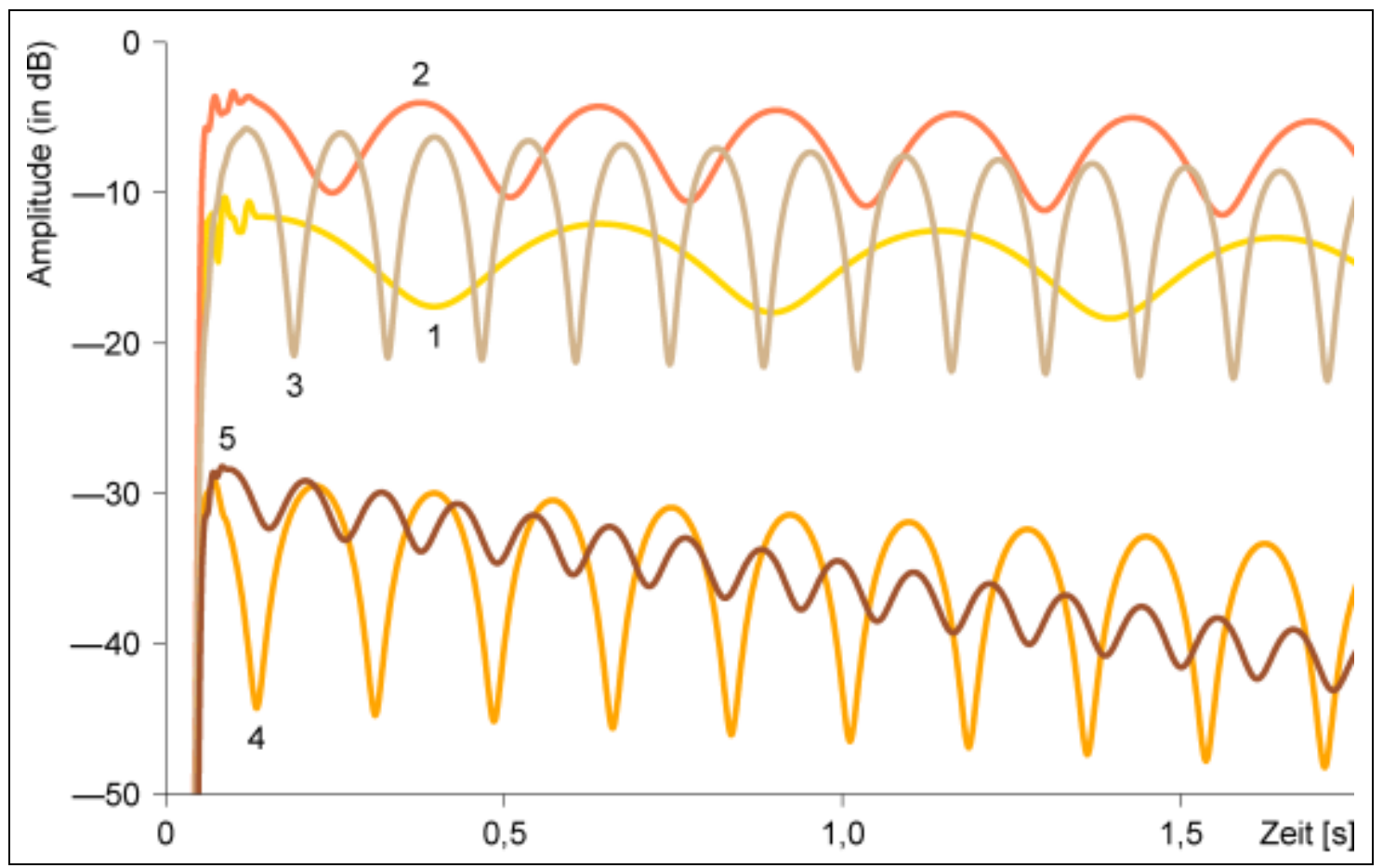

Abb. 2: Zu Klangbeispiel 2, Pegelverläufe der synthetisierten Teilschwingungen (linker Kanal)

Neben dem Vergleich des ersten Klangs mit einem Klang, der sich rascher bewegt, soll der Hörversuch einen Vergleich mit einem ruhiger bewegten Klang enthalten. Für das Verringern der Schwebungsfrequenz bietet sich die dritte Teilschwingung an. Die unteren drei Teilschwingungen sind über lange Zeit gut hörbar; unter ihnen schwebt die dritte am schnellsten. Als Variante wurde ihre Schwebungsfrequenz halbiert (Klangbeispiel 3, Abb. 3). Das Ergebnis unterscheidet sich weniger stark vom ersten Klang als die vorangegangene Variante (Klangbeispiel 2). 
Klangbeispiel 3: Wie Klangbeispiel 1, aber Schwebungsfrequenz der dritten Teilschwingung halbiert (Klang_3.mp3)

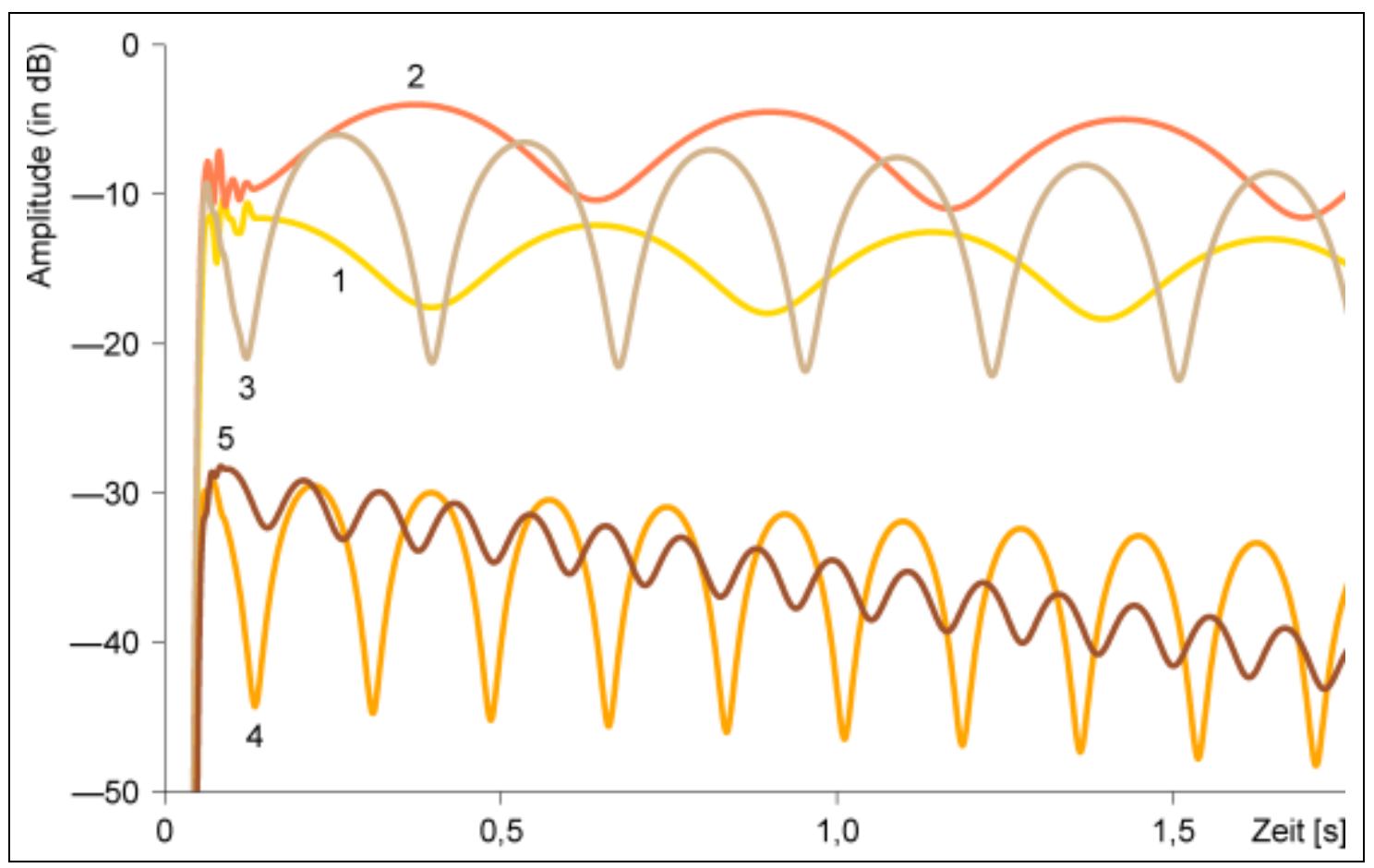

Abb. 3: Zu Klangbeispiel 3, Pegelverläufe der synthetisierten Teilschwingungen (linker Kanal)

Eine schwebungsfreie Klangschale wird man kaum finden, denn sie müsste genau rotationssymmetrisch sein. Messungen an einigen gedrehten japanischen Schalen ergaben Schwebungen mit niedriger Frequenz (Thies 2011, S. 36). Um die Reaktion der Versuchsteilnehmer auf einen Klang festzustellen, der ohne Fluktuation allmählich verebbt, ist diese Variante dennoch einbezogen (Klangbeispiel 4).

Klangbeispiel 4: Wie Klangbeispiel 1, aber alle Teilschwingungen schwebungsfrei (Klang_4.mp3) ๑

Für den Hörvergleich einer mittelgroßen und einer kleinen Schale ist eine weitere Variante des zuerst synthetisierten Audiosignals nötig (s. unter 3.3, Klangbeispiel 6). Eine andere Variante dient dem Vergleich einer mittelgroßen und einer großen Schale (s. unter 3.3, Klangbeispiel 8, Abb. 5). Um ein zusätzliches Vergleichspaar mit verschieden schneller Bewegung der Klänge $\mathrm{zu}$ erhalten, wurde die letztgenannte Variante ihrerseits variiert. Auch diese Abwandlung erscheint im Abschnitt „Variation der Schalengröße“, um den Vergleich mit Klangbeispiel 8 zu erleichtern (Klangbeispiel 10, Abb. 6). 


\subsection{Variation der Schalengröße}

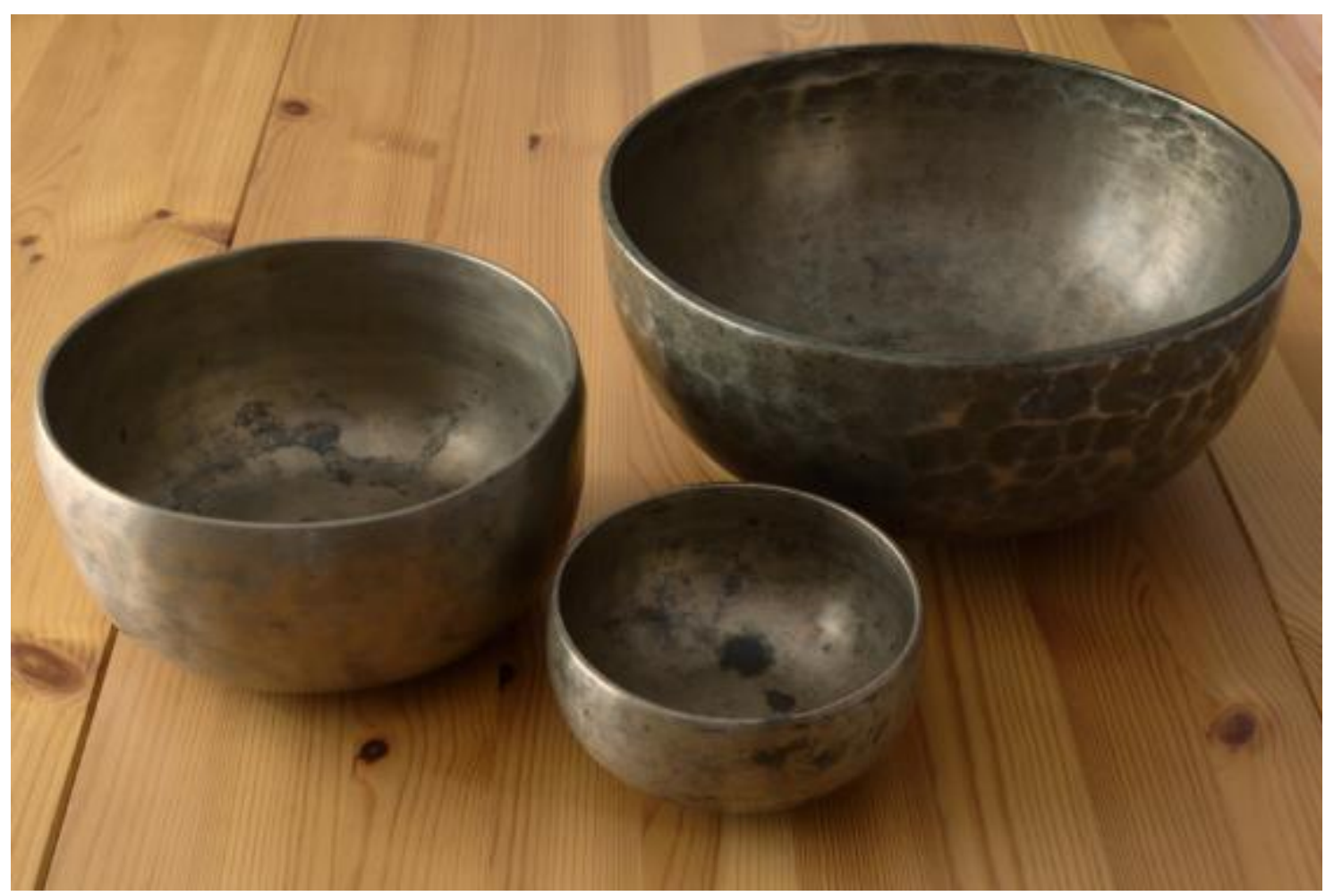

Abb. 4: Drei Klangschalen, die ungefähr im Oktavabstand klingen

(Durchmesser $17 \mathrm{~cm}, 11 \mathrm{~cm}, 24 \mathrm{~cm}$ )

Um den Klang der mittelgroßen Schale mit Klängen einer kleinen und einer großen Schale vergleichen $\mathrm{zu}$ können, wurden weitere Schalenschwingungen synthetisiert. Die Schwingungsfrequenzen waren gegenüber der mittelgroßen Schale verdoppelt bzw. halbiert. Realistisch klingen diese Oktavtranspositionen aber nur dann, wenn sie die Eigenheiten kleinerer bzw. größerer Schalen einbeziehen. Mit abnehmender Schalengröße ist die Schwingung stärker gedämpft, und die Amplitude der Teilschwingungen fällt zu den hohen Frequenzen hin stärker ab. Man muss also mehr Schalleigenschaften ändern als nur die Frequenzen der Teilschwingungen. Als Vorbild hierfür dienten Klangschalen, die ungefähr im Oktavabstand zur mittelgroßen Schale klingen, ebenfalls dickwandig sind und ähnlich angeschlagen wurden wie die mittelgroße Schale (Abb. 4).

Wenn man die kleine Schale in dieser Weise anschlägt und aus einer ähnlichen Position hört wie im Abschnitt 3.2 für die mittelgroße Schale beschrieben, sind drei Teiltöne wahrnehmbar. Um aus den Synthesedaten der mittelgroßen Schale Daten für eine kleine Schale zu erhalten und nicht mehr als nötig einzugreifen, bietet sich das folgende Verfahren an: Bei den Teilschwingungen 1-3 werden die Frequenzen der zwei 
Komponenten, in die jede Teilschwingung aufgespalten ist, so erhöht, dass ihr Mittelwert sich verdoppelt, während ihre Differenz (und damit die Schwebungsfrequenz) gleich bleibt; die Amplitudenverhältnisse beim Anschlag und die Dämpfung der Teilschwingungen werden entsprechend den Beobachtungen an der kleinen Schale eingestellt; die Teilschwingungen 4 und 5 entfallen (Klangbeispiel 5).

Klangbeispiel 5: Wie Klangbeispiel 1, aber mit den beschriebenen Änderungen für eine kleine Schale (klein.mp3)

Im Vergleich mit dem Ausgangsklang (Klangbeispiel 1) erscheint der höhere Klang weniger räumlich. Dieser Unterschied ist bei live gespielten Schalen mit entsprechendem Größenverhältnis ebenfalls $\mathrm{zu}$ beobachten und sollte im Interesse realitätsnaher Klänge erhalten bleiben. Die simulierte kleine Schale klingt aber auch weniger bewegt als die mittelgroße. Dies ist nicht erwünscht, denn die im Hörversuch zu vergleichenden Klänge sollen sich möglichst nur durch Eigenschaften unterscheiden, die mit der Größe der Schalen zusammenhängen.

Damit der Klang der mittelgroßen Schale und der transponierte Klang ein ähnliches Bewegungsmuster zeigen, sind weitere Überlegungen nötig. Wie kommt die langsamere Bewegung des höheren Klangs zustande? Bei der mittelgroßen Schale stehen die Teilschwingungen 1-3 klanglich im Vordergrund. Ihre Schwebungsfrequenzen sind 2,0 Hz, 1,9 Hz und 7,2 Hz. Bei der kleinen, ähnlich angeschlagenen Schale treten nur die ersten beiden Teilschwingungen hervor, die dritte ist schwach ausgeprägt und verschwindet nach wenigen Sekunden. Wenn man für die Synthese die Schwebungsfrequenzen der mittelgroßen Schale übernimmt, ist der Klang langsamer bewegt, weil die Teilschwingung mit der Schwebungsfrequenz 7,2 Hz hier weit in den Hintergrund tritt. Ungünstig ist ferner, dass man bei den klangbeherrschenden Teiltönen der Transposition Schwebungen mit nahezu gleicher Frequenz (2,0 und 1,9 Hz) hört, die etwas aufdringlich wirken können; beim tieferen Klang lockert die zusätzliche Schwebungsfrequenz 7,2 Hz das Bewegungsmuster auf.

Um für den Paarvergleich im Hörversuch zwei Klänge verschieden großer Schalen mit ähnlichem Bewegungsmuster zu erhalten, wird eine Variante des zuerst synthetisierten Audiosignals verwendet. Hier unterscheiden sich die Schwebungsfrequenzen der Teilschwingungen 1 und 2 stärker $(2,4$ und 1,4 Hz), die Schwebungsfrequenz der dritten Teilschwingung ist niedriger $(1,95 \mathrm{~Hz})$. Diese drei Schwebungsfrequenzen sind bei der mittelgroßen Schale am deutlichsten hörbar, ihr Mittelwert beträgt 1,92 Hz. Bei der eine Oktave höher klingenden Schale stehen die 
Teilschwingungen 1 und 2 im Vordergrund, der Mittelwert der Schwebungsfrequenzen ist mit $1,90 \mathrm{~Hz}$ nahezu gleich. Gleiche Differenzen von Schwebungsfrequenz zu Schwebungsfrequenz und einfache Frequenzverhältnisse treten nicht auf (hierdurch können Bewegungsmuster entstehen, die sich in kurzen Zeitabständen wiederholen). Die akustischen Eigenschaften des Vergleichspaars lauten somit: Die Variante des ursprünglichen Signals (Klangbeispiel 1) hat geänderte Schwebungsfrequenzen der Teilschwingungen 1-3 (2,4 Hz, 1,4 Hz, 1,95 Hz), die übrigen Synthesedaten bleiben gleich (Klangbeispiel 6). Gegenüber dieser Variante sind bei der Nachahmung einer kleinen Schale für jede der Teilschwingungen 1-3 die Frequenzen der beiden Komponenten so erhöht, dass sich ihr Mittelwert verdoppelt, während ihre Differenz (und damit die Schwebungsfrequenz) gleich bleibt. Die Amplitudenverhältnisse und Dämpfungswerte der Teilschwingungen 1-3 sind hier der geringeren Schalengröße angepasst, die Teilschwingungen 4 und 5 entfallen. Die übrigen Synthesedaten bleiben gleich (Klangbeispiel 7).

Klangbeispiel 6: Wie Klangbeispiel 1, aber Schwebungsfrequenzen der Teilschwingungen 1-3 2,4 Hz, 1,4 Hz, 1,95 Hz (Klang_6.mp3) ๑

Klangbeispiel 7: Wie Klangbeispiel 6, aber bei den Teilschwingungen 1-3 Frequenzen der beiden Komponenten so erhöht, dass ihr Mittelwert doppelt so groß ist und ihre Differenz gleich bleibt; Amplitudenverhältnisse und Dämpfung der Teilschwingungen 1-3 der geringeren Schalengröße angepasst; Teilschwingungen 4 und 5 entfernt (Klang_7.mp3)

Um aus den Synthesedaten der mittelgroßen Schale Daten für eine große Schale abzuleiten, sind wiederum die Amplitudenverhältnisse und die Dämpfung der Teilschwingungen anzupassen. Die als Vorbild genutzte, ebenfalls dickwandige Schale (rechts in Abb. 4), die ungefähr eine Oktave tiefer klingt, muss etwas sanfter angeschlagen werden als die mittelgroße Schale, damit sie ähnlich laut klingt wie jene. Unter diesen Bedingungen ist ein sechster Teilton kurz hörbar, daher tritt bei der Synthese eine weitere Teilschwingung hinzu.

Wie beim zuvor beschriebenen Vergleichspaar sind weitere Kriterien zu berücksichtigen, damit der Klang der mittelgroßen Schale und der transponierte Klang ein ähnliches Bewegungsmuster zeigen. Bei der mittelgroßen Schale treten die Teilschwingungen 1-3 klanglich hervor, ihre Schwebungsfrequenzen sind 2,0 Hz, 1,9 Hz und 7,2 Hz. Bei der großen, etwas sanfter angeschlagenen Schale stehen die Teilschwingungen 1-4 im Vordergrund. Wenn man für die Synthese die 
Schwebungsfrequenzen der mittelgroßen Schale übernimmt, ist der Klang bewegter, denn die bei jener Schale unauffällige vierte Teilschwingung ist jetzt ebenfalls kräftig und lang hörbar. Ihre Schwebungsfrequenz 5,7 Hz beschleunigt die Klangbewegung. Noch wichtiger ist wahrscheinlich, dass der dritte Teilton der Transposition nahe am zweiten Teilton des Ausgangsklangs liegt (einen Halbton tiefer): Wenn an dieser Stelle des Teiltonaufbaus die Schwebungsfrequenz von 1,9 Hz auf 7,2 $\mathrm{Hz}$ springt, erscheint dies als erhebliche Steigerung der Klangbewegung.

Um für den Paarvergleich im Hörversuch zwei Klänge verschieden großer Schalen mit ähnlichem Bewegungsmuster zu erhalten, ist eine weitere Variante des zuerst hergestellten Audiosignals nötig. Hier sind die Schwebungsfrequenzen so gewählt, dass sich die beiden zu vergleichenden Klänge ähnlich ruhig bewegen. Die Schwebungsfrequenzen der Teilschwingungen 1-3 entsprechen jenen im Vergleich von mittelgroßer und kleiner Schale $(2,4 \mathrm{~Hz}, 1,4 \mathrm{~Hz}, 1,95 \mathrm{~Hz})$, damit für den Klangvergleich bei der Transposition nach oben und nach unten zwei Klangpaare mit ähnlicher Bewegung zur Verfügung stehen. Die Schwebungsfrequenz der vierten Teilschwingung beträgt 2,06 Hz. Somit haben die Schwebungsfrequenzen bei den klanglich hervortretenden Teilschwingungen 1-3 der mittelgroßen Schale und bei den hervortretenden Teilschwingungen 1-4 der großen Schale nahezu den gleichen Mittelwert (1,92 Hz bzw. 1,95 Hz). Gleiche Differenzen von Schwebungsfrequenz zu Schwebungsfrequenz und einfache Frequenzverhältnisse kommen nicht vor, um Bewegungsmuster zu vermeiden, die sich in kurzen Zeitabständen wiederholen. Die akustischen Eigenschaften des Vergleichspaars lauten daher: Die Variante des ursprünglichen Audiosignals (Klangbeispiel 1) hat geänderte Schwebungsfrequenzen der Teilschwingungen 1-4 $(2,4 \mathrm{~Hz}, 1,4 \mathrm{~Hz}, 1,95 \mathrm{~Hz}, 2,06 \mathrm{~Hz})$, die übrigen Synthesedaten bleiben gleich (Klangbeispiel 8). Gegenüber dieser Variante sind bei der Nachahmung einer großen Schale für die Teilschwingungen 1-5 die Frequenzen der beiden Komponenten, in die jede Teilschwingung aufgespalten ist, so erniedrigt, dass sich ihr Mittelwert halbiert, während ihre Differenz (und damit die Schwebungsfrequenz) gleich bleibt. Die Amplitudenverhältnisse und Dämpfungswerte der Teilschwingungen 1-5 sind dem größeren Schalendurchmesser angepasst. Eine sechste Teilschwingung wird so synthetisiert, dass das Frequenzverhältnis zur fünften Teilschwingung, das Amplitudenverhältnis und die Dämpfung ebenso groß sind wie bei der als Vorbild gewählten großen Schale. Schwebungsfrequenz und Schwebungshub entsprechen den Durchschnittswerten für die übrigen synthetisierten Teilschwingungen. Alle anderen Synthesedaten bleiben gleich (Klangbeispiel 9). Die Nachahmung der 
großen Schale klingt etwas räumlicher, dies entspricht dem Eindruck bei der live gespielten Schale.

Klangbeispiel 8: Wie Klangbeispiel 1, aber Schwebungsfrequenzen der Teilschwingungen 1-4 2,4 Hz, 1,4 Hz, 1,95 Hz, 2,06 Hz (Klang_8.mp3)

Klangbeispiel 9: Wie Klangbeispiel 8, aber bei den Teilschwingungen 1-5 Frequenzen der beiden Komponenten so erniedrigt, dass ihr Mittelwert halb so groß ist und ihre Differenz gleich bleibt; Amplitudenverhältnisse und Dämpfung der Teilschwingungen 1-5 dem größeren Schalendurchmesser angepasst; Teilschwingung 6 hinzugefügt (Klang_9.mp3)

Die für diesen Paarvergleich hergestellte Variante des zuerst hergestellten Audiosignals hat unter den schwebungshaltigen Varianten die niedrigsten Schwebungsfrequenzen (Klangbeispiel 8, Abb. 5). Um zusätzlich zu Klang_1 und Klang_2 (Klangbeispiele 1 und 2) ein ergänzendes Vergleichspaar zu erhalten, in dem der zweite Klang rascher bewegt ist, wurde aus dieser Variante eine weitere Variante abgeleitet: Hier sind die Schwebungsfrequenzen der Teilschwingungen 2 und 3 verdoppelt (Klangbeispiel 10, Abb. 6). Diese zum Thema „Variation der Schwebungsfrequenz" gehörende Variante steht hier, um den Vergleich mit Klangbeispiel $8 \mathrm{zu}$ erleichtern.

Klangbeispiel 10: Wie Klangbeispiel 8, aber Schwebungsfrequenzen der Teilschwingungen 2 und 3 verdoppelt (Klang_10.mp3) 


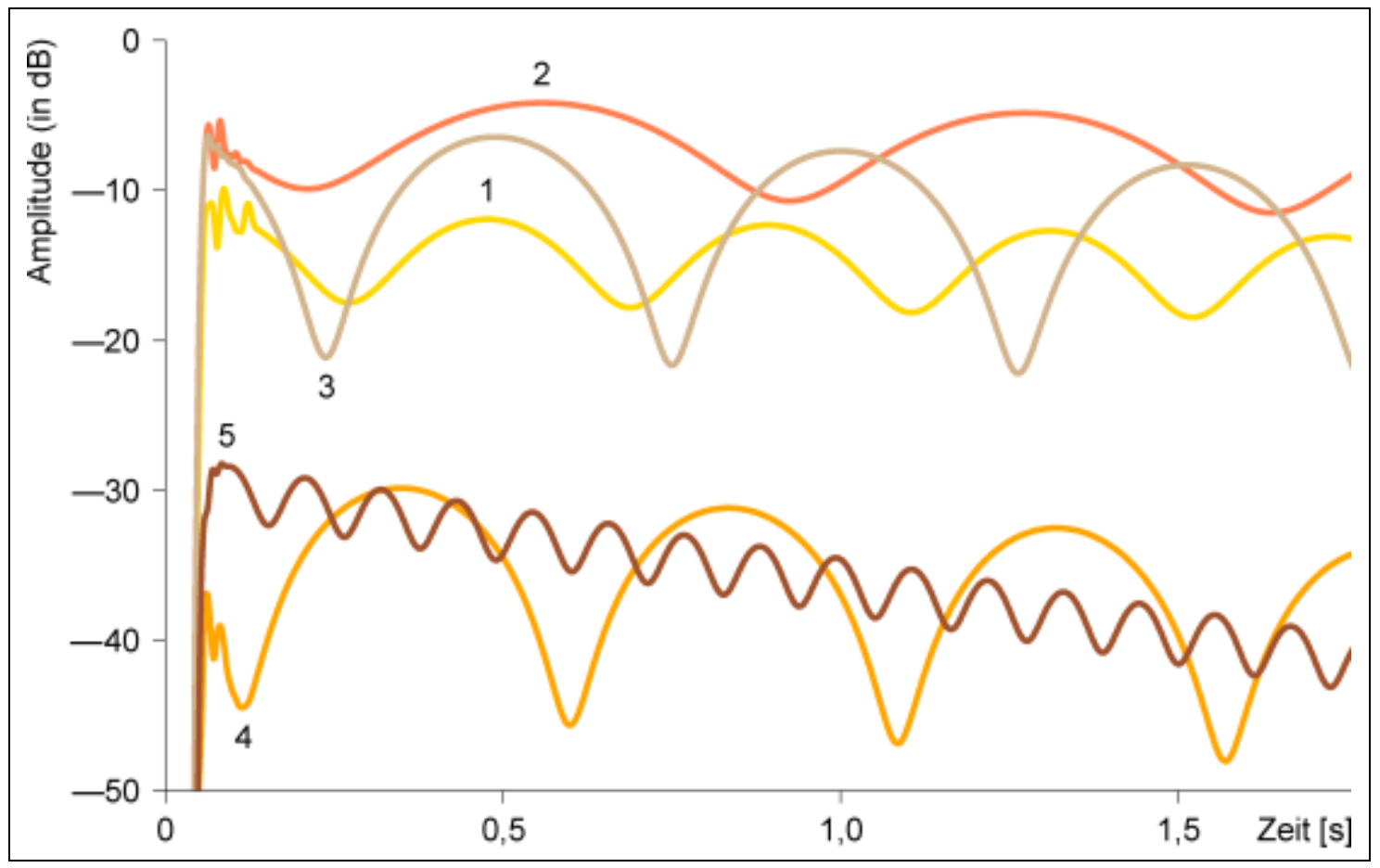

Abb. 5: Zu Klangbeispiel 8, Pegelverläufe der synthetisierten Teilschwingungen (linker Kanal)

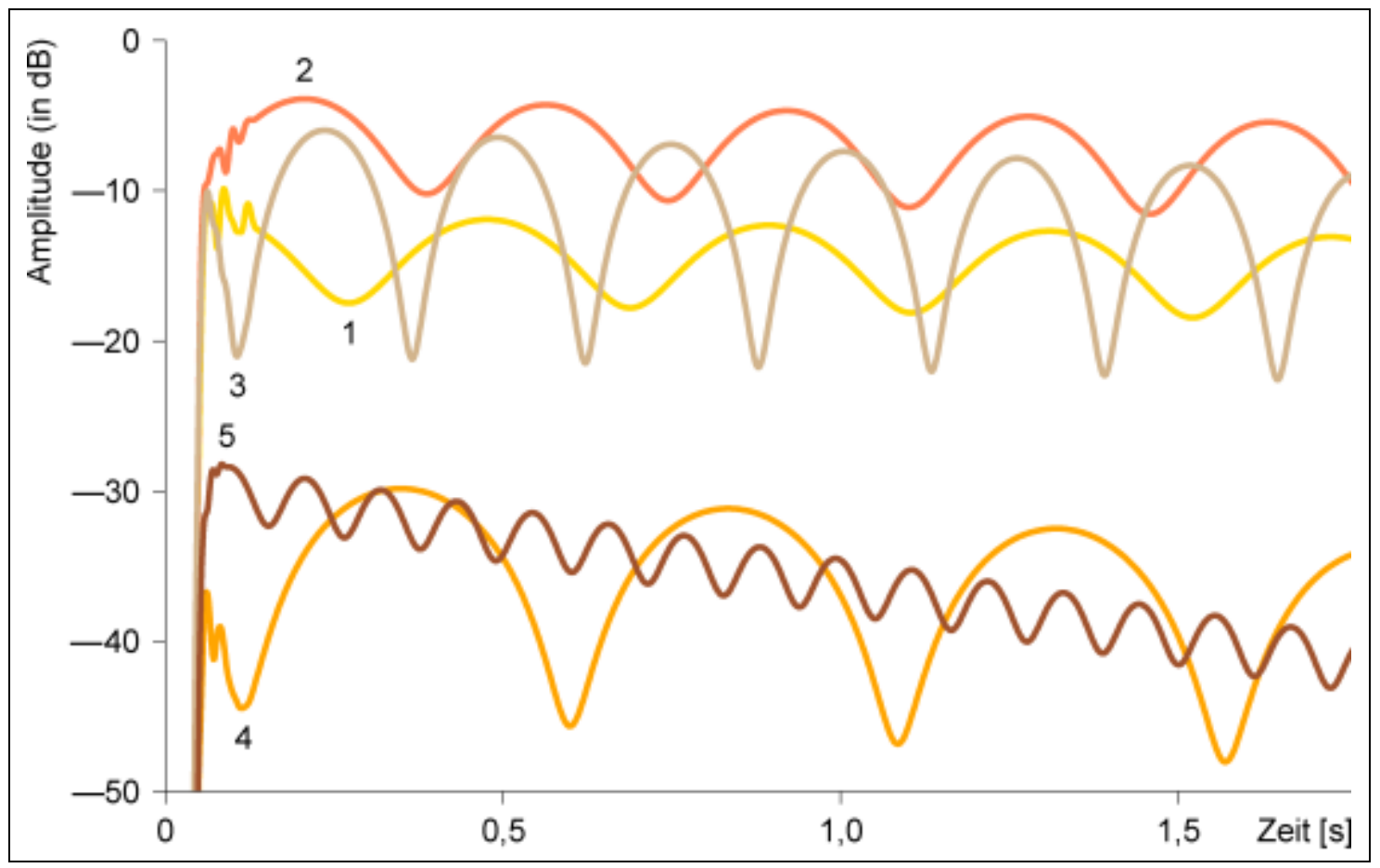

Abb. 6: Zu Klangbeispiel 10, Pegelverläufe der synthetisierten Teilschwingungen (linker Kanal) 


\subsection{Anordnung der Audiosignale im Hörversuch}

Im Hörversuch sollen die Teilnehmer Audiosignale paarweise vergleichen. Damit die Hörer nicht ermüden oder das Tragen des Kopfhörers lästig wird, ist die Anzahl der Paarvergleiche auf sechs begrenzt. Die Paare sind der Reihe nach anzuhören. Vier Klangpaare $(1,3,5,6)$ zeigen Unterschiede der Klangbewegung bei gleicher Tonlage. Der feinste Unterschied der Bewegung erscheint im ersten Klangpaar, der auffälligste Unterschied im letzten, um nicht von vornherein die Aufmerksamkeit auf die Klangbewegung zu lenken. Bei Klangpaar 1 ist der erste Klang rascher bewegt, bei Klangpaar 3 der zweite Klang. Bei den Klangpaaren 5 und 6 ist die Reihenfolge umgekehrt: In Paar 5 hat der zweite Klang mehr Bewegung, in Paar 6 der erste. Der erste Klang der Paare 1 und 3 ist schneller bewegt als der erste Klang in den Paaren 5 und 6. Die Klangpaare 2 und 4 zeigen verschiedene Tonlage bei ähnlicher Beweg theit. Sie sind zwischen Paare mit verschiedener Bewegung gestellt, um Abwechslung zu erreichen und wiederum die Aufmerksamkeit nicht zu sehr auf die Bewegung des Klangs zu konzentrieren. Beim sechsten Klangpaar schließlich ist der Unterschied der Klangbewegung unüberhörbar: Der letzte Klang verebbt gleichmäßig, ohne zu fluktuieren.

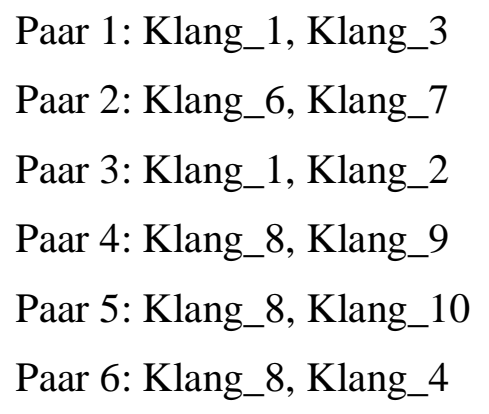

\section{Der Fragebogen für den Hörversuch}

Der Fragebogen für die Versuchsteilnehmer beginnt mit einer Anleitung (Abb. 7). Der Kasten mit den Fragen sieht bei den Klangpaaren 2, 4, 5 und 6 genauso aus wie in der Anleitung (Abb. 8). Die Klänge der Paare 1 und 3 unterscheiden sich am wenigsten; deswegen wird dort ergänzend nach dem gehörten Unterschied gefragt, sofern die Antwort auf die zweite Frage ,gleichgültig“ lautet (Abb. 9). Der Hinweis bei diesen beiden Paaren, es gehe nicht um die Prüfung des Hörvermögens, erscheint wichtig, weil viele Teilnehmer den zuvor durchgeführten Versuch (Thies 2008) als „Hörtest“ bezeichnet haben. Die Beteiligten sollen keinen Stress empfinden in der Annahme, möglicherweise „falsch“ zu antworten. Die durch Ankreuzen gegebenen Antworten 
erläutern die Teilnehmer mündlich, um spontane Äußerungen zu erleichtern. Die mündlichen Auskünfte entfallen bei der Antwortfolge ,ja“-,,gleichgültig“ (bei den Klangpaaren 1 und 3: ,ja“"-,,gleichgültig“-,,nein“).

Bitte hören Sie sich sechs Klangpaare an und vergleichen Sie jeweils die beiden Klänge.

Nach dem Anhören jedes Paars werden Ihnen einige Fragen gestellt:

Nehmen wir an, Sie möchten sich entspannen. Meinen Sie, dass einer der Klänge (oder beide) Sie dabei unterstützen könnte?

\begin{tabular}{|c|c|c|c|}
\hline \multicolumn{3}{|c|}{$\square$ ja } & $\square$ nein \\
\hline \multicolumn{3}{|c|}{ Welchen Klang würden Sie zum Entspannen lieber hören? } & \multirow{3}{*}{$\begin{array}{l}\text { Bitte nennen } \\
\text { Sie (mündlich) } \\
\text { den Grund. }\end{array}$} \\
\hline$\square 1$ & $\square 2$ & \multirow[t]{2}{*}{$\square$ gleichgültig } & \\
\hline \multicolumn{2}{|c|}{$\begin{array}{l}\text { Bitte nennen Sie (mündlich) der } \\
\text { Grund für lhre Wahl. }\end{array}$} & & \\
\hline
\end{tabular}

Bei zwei Klangpaaren wird außerdem gefragt, welche Klangunterschiede Sie gehört haben. Dabei geht es nicht um die Prüfung Ihres Hörvermögens, sondern allein um Ihre persönlichen Eindrücke.

*

Zum Abspielen eines Klangs brauchen Sie nur den Zeiger auf das Symbol zu bringen und die linke Maustaste einmal zu drücken. Wenn Sie erneut drücken, beginnt der Klang wieder von vorn. Jeder Klang dauert etwa eine Minute. Hören Sie so lange zu, wie Sie möchten. Es steht Ihnen frei, von vorn zu beginnen oder zum anderen Klang eines Paares zu wechseln, bevor ein Klang zu Ende ist.

Um zum anderen Klang zu wechseln, bringen Sie einfach den Zeiger auf das andere Symbol und drücken wieder die linke Maustaste. Wechseln Sie zwischen den Klängen eines Paars, bis Sie zum Beantworten der Fragen bereit sind.

Wenn die Lautstärke für Sie nicht angenehm ist, sagen Sie bitte Bescheid.

Abb. 7: Versuchsanleitung 


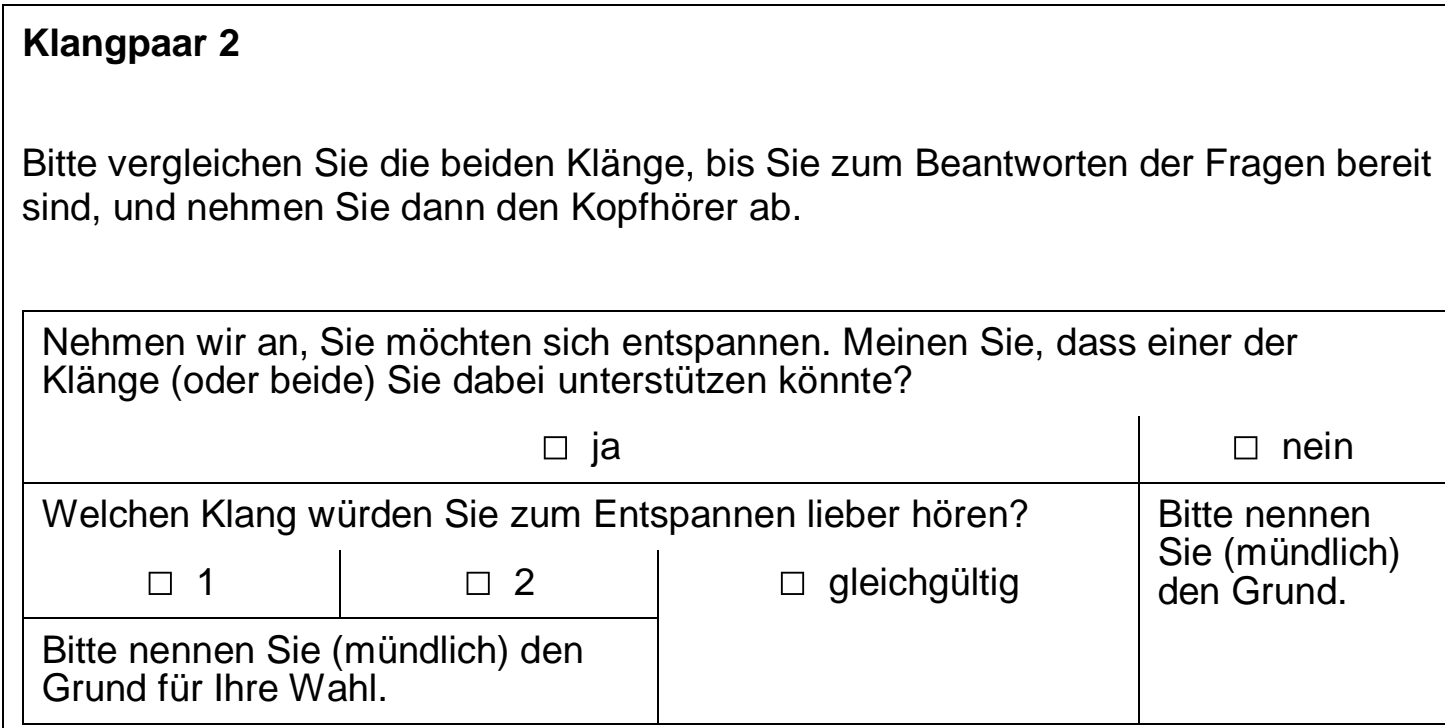

Abb. 8: Fragen bei den Klangpaaren 2, 4, 5 und 6

\section{Klangpaar 3}

Bitte vergleichen Sie die beiden Klänge, bis Sie zum Beantworten der Fragen bereit sind, und nehmen Sie dann den Kopfhörer ab.

Es geht nicht um die Prüfung Ihres Hörvermögens, sondern allein um Ihre persönlichen Eindrücke. Insofern gibt es keine „richtigen“ oder „falschen“ Antworten, Sie können sich völlig frei äußern.

Nehmen wir an, Sie möchten sich entspannen. Meinen Sie, dass einer der Klänge (oder beide) Sie dabei unterstützen könnte?

\begin{tabular}{|c|c|c|c|c|}
\hline \multicolumn{4}{|c|}{$\square$ ja } & $\square$ nein \\
\hline \multicolumn{4}{|c|}{ Welchen Klang würden Sie zum Entspannen lieber hören? } & \multirow{5}{*}{$\begin{array}{l}\text { Bitte nennen } \\
\text { Sie (mündlich) } \\
\text { den Grund. }\end{array}$} \\
\hline$\square 1$ & $\square 2$ & $\square$ gleichgi & Itig & \\
\hline \multirow{3}{*}{\multicolumn{2}{|c|}{$\begin{array}{l}\text { Bitte nennen Sie (mündlich) den } \\
\text { Grund für Ihre Wahl. }\end{array}$}} & \multicolumn{2}{|c|}{$\begin{array}{l}\text { Haben Sie einen Klang- } \\
\text { unterschied gehört? }\end{array}$} & \\
\hline & & $\square$ ja & \multirow[t]{2}{*}{$\square$ nein } & \\
\hline & & $\begin{array}{l}\text { Bitte erzählen } \\
\text { Sie, welchen } \\
\text { Unterschied Sie } \\
\text { gehört haben. }\end{array}$ & & \\
\hline
\end{tabular}

Abb. 9: Fragen bei den Klangpaaren 1 und 3 


\section{Versuchspersonen}

Nachdem ein früherer Hörversuch (Thies 2008) gezeigt hat, dass das Hören einer Klangschale eine gute Entspannungshilfe sein kann, steht dieses Thema jetzt im Mittelpunkt. Für diesen Versuch kommen somit nur Menschen in Frage, die grundsätzlich der Ansicht sind, Schalenklänge könnten sie beim Entspannen unterstützen. Die Auswahl konzentrierte sich daher zunächst auf Personen, die im früheren Versuch die Klänge als Entspannungshilfe empfunden hatten. Die ergänzend zur Teilnahme eingeladenen Personen bekamen, sofern sie bereit und Klangschalen ihnen unbekannt waren, einen Probeklang über Kopfhörer zu hören (Klang_3, Klangbeispiel 3). Sie wurden gefragt, ob sie meinen, sich mit derartigen Klängen entspannen zu können. $15 \%$ von ihnen empfanden den Klang nicht als angenehm, daher schieden sie aus.

Am Hörversuch beteiligten sich 31 Frauen und 29 Männer im Alter von 24 bis 75 Jahren (Median 44 Jahre, $85 \%$ in der Altersgruppe 30 bis 60 Jahre). Die Berufe waren breit gestreut, einen Musikberuf hatten nur zwei Teilnehmer (Instrumentallehrer, Instrumentenbauer). Eine Beeinflussung der Versuchsergebnisse durch einen hohen Anteil von Personen mit außergewöhnlich geschultem Gehör ist daher nicht zu erwarten.

\section{Versuchsdurchführung}

Der Hörversuch fand in Einzelsitzungen statt. Die Teilnehmer saßen auf einem bequemen Stuhl an einem Tisch, vor ihnen stand ein Notebook mit angeschlossener Maus zum Abspielen der Audiosignale. Die Schallimmission von außen war gering. Ein geschlossener Kopfhörer mit guter Dämpfung des Umgebungsschalls sorgte dafür, dass die Klänge in Ruhe angehört werden konnten. Richtschnur für die Lautstärkeeinstellung war eine reale, mittelstark angeschlagene Klangschale. Wenn jemandem dies zu laut erschien, wurde die Einstellung gesenkt.

$\mathrm{Zu}$ Beginn erhielt jede Versuchsperson einen Fragebogen mit Anleitung (s. unter 4). Bei Bedarf gab der Versuchsleiter weitere Auskünfte zum Ablauf. Danach begann das Hören der Klänge. Bei jedem der sechs Klangpaare (s. unter 3.4) erschienen auf dem Bildschirm zwei Symbole für die zu vergleichenden Klänge. Ein einfacher Mausklick auf ein Symbol startete das Abspielen. Ohne das Verklingen abzuwarten (bei der simulierten mittelgroßen Schale nach etwa einer Minute), konnte man durch erneuten Klick den Klang wieder von vorn abspielen oder zum anderen Klang 
wechseln. Als Zeitpuffer nach dem Mausklick begann jede Audiodatei mit einer Pause von 0,6 Sekunden. Für den Klangvergleich gab es keine Zeitgrenze. Danach legte die Versuchsperson den Kopfhörer ab und beantwortete die Fragen durch Ankreuzen. Je nachdem, welche Antworten sie gab, folgten möglicherweise mündliche Erläuterungen. Der Versuchsleiter hatte Gelegenheit, durch Nachfragen weitere Auskünfte zu erhalten. Er protokollierte die mündlichen Mitteilungen stichwortartig während des Gesprächs.

\section{Auswertung des Hörversuchs}

\subsection{Alle Klangpaare, erste Frage}

Der Hörversuch umfasste 360 Paarvergleiche von Klängen (sechs Klangpaare, 60 Personen). Nach jedem Vergleich lautete die erste Frage: „Nehmen wir an, Sie möchten sich entspannen. Meinen Sie, dass einer der Klänge (oder beide) Sie dabei unterstützen könnte?“ $84 \%$ der Antworten lauteten ,ja“. Die meisten Teilnehmer antworteten bei allen sechs Klangpaaren mit ,ja“, niemand bejahte die Frage bei weniger als zwei Klangpaaren (Tab. 1).

\begin{tabular}{|lccccccc|}
\hline $\begin{array}{l}\text { Anzahl der Klangpaare } \\
\text { mit der Antwort ,ja“ }\end{array}$ & 0 & 1 & 2 & 3 & 4 & 5 & 6 \\
Anzahl der Hörer & $0 \%$ & $0 \%$ & $8 \%$ & $8 \%$ & $12 \%$ & $12 \%$ & $60 \%$ \\
\hline
\end{tabular}

Tab. 1: Antwort ,ja“ auf die erste Frage bei allen Klangpaaren

\subsection{Klangpaar 1: Klang_1, Klang_3}

Bei gleicher Tonlage unterscheiden sich die Klänge durch ihre Bewegung. Im zweiten Klang fluktuiert der dritte Teilton halb so schnell. Unter den vier Klangpaaren mit verschiedener Bewegtheit der Klänge zeigt dieses Paar den geringsten Unterschied. Die Häufigkeiten der Antworten auf die einzelnen Fragen sind in Tab. 2 zusammengestellt. Die Prozentwerte beziehen sich hier und in den Tabellen für die anderen Klangpaare jeweils auf die Gesamtheit bzw. die Untergruppe der Teilnehmer: bei der ersten Frage auf alle Hörer, bei der zweiten Frage auf jene, welche die erste Frage bejahten und bei der dritten Frage auf jene, die mit ,gleichgültig“ antworteten. 


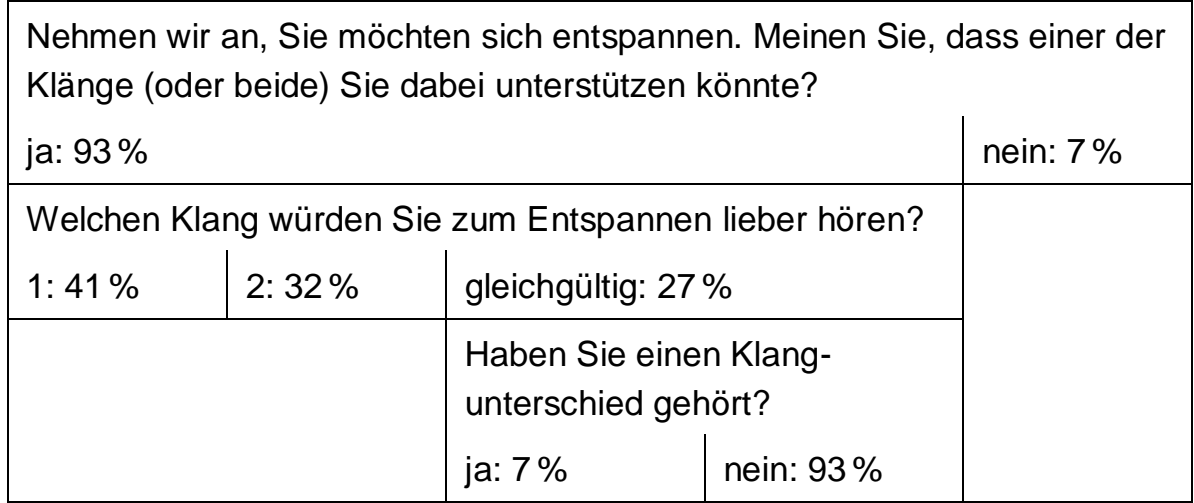

Tab. 2: Antworten bei Klangpaar 1

Die Antwort „nein“ auf die erste Frage begründete die Hälfte der Hörer mit dem Oszillieren, den übrigen waren die Klänge zu hoch.

Auf die zweite Frage wurden für das Bevorzugen eines Klangs viele verschiedene Gründe genannt, die teilweise beim ersten und zweiten Klang übereinstimmen - auch bei Aussagen zur Klangbewegung. Beispielsweise gab es zum ersten Klang Bemerkungen wie „Wellen langsamer“, „pulsiert schwächer“ und „Lautstärkeschwankungen bei höheren Tönen ruhiger“; beim zweiten Klang kamen die Kommentare „langsamere Schwingung“ und „Oberfrequenz langsamer“ vor. Von jenen Hörern, die den ersten Klang bevorzugten, bezeichneten $22 \%$ den Unterschied als sehr gering. (In der Gruppe, die den zweiten Klang wählte, gab es keinen derartigen Hinweis.)

Bei der Antwort „gleichgültig“ hörte nur eine Person einen Klangunterschied. Sie beschrieb ihn als „marginal“ (zweiter Klang etwas dunkler, Schwingen setzt später ein).

\subsection{Klangpaar 3: Klang_1, Klang_2}

Beim zweiten Paar mit verschiedener Bewegtheit der Klänge ist der zweite Klang schneller bewegt. Der erste Klang entspricht dem ersten Klang im ersten Klangpaar. Im zweiten Klang fluktuiert der zweite Teilton doppelt so schnell. Dies ist der lauteste Teilton, daher sollte der Unterschied beider Klänge gut hörbar sein (Ergebnisse s. Tab. 3). 


\begin{tabular}{|c|c|c|c|c|}
\hline \multicolumn{5}{|c|}{$\begin{array}{l}\text { Nehmen wir an, Sie möchten sich entspannen. Meinen Sie, dass einer der } \\
\text { Klänge (oder beide) Sie dabei unterstützen könnte? }\end{array}$} \\
\hline \multicolumn{4}{|c|}{ Welchen Klang würden Sie zum Entspannen lieber hören? } & \\
\hline $1: 71 \%$ & $2: 16 \%$ & \multicolumn{2}{|c|}{ gleichgültig: $13 \%$} & \\
\hline & & \multicolumn{2}{|c|}{$\begin{array}{l}\text { Haben Sie einen Klang- } \\
\text { unterschied gehört? }\end{array}$} & \\
\hline & & ja: $33 \%$ & nein: $67 \%$ & \\
\hline
\end{tabular}

Tab. 3: Antworten bei Klangpaar 3

Von den Hörern, die mit „nein“ antworteten, empfanden $67 \%$ die Bewegung der Klänge als zu stark. Andere Teilnehmer (20\%) nannten die hohe Lage als Grund.

Teilnehmer, die bei der zweiten Frage den ersten Klang wählten, begründeten dies zu $88 \%$ mit der geringeren Bewegung. In der Gruppe, die sich für den zweiten Klang entschied, wurde die intensivere Bewegung als häufigster Grund genannt (57\%); andere Klangeigenschaften treten demgegenüber in den Hintergrund. Von den Personen, die mit „gleichgültig“ antworteten, hörten zwei einen Klangunterschied (Volumen bzw. Höhe).

$68 \%$ aller Teilnehmer wiesen auf stärkere Bewegung des zweiten Klangs hin.

\subsection{Klangpaar 5: Klang_8, Klang_10}

Auch hier ist der zweite Klang schneller bewegt als der erste. Der erste Klang fluktuiert langsamer als der erste Klang in den Paaren 1 und 3. Unter allen Klängen der Paare 1, 3 und 5 hat er die geringste Bewegtheit. Im zweiten Klang fluktuieren der zweite und der dritte Teilton doppelt so schnell. Tab. 4 zeigt die Häufigkeitsverteilung der Antworten.

\begin{tabular}{|c|c|c|c|}
\hline \multicolumn{4}{|c|}{$\begin{array}{l}\text { Nehmen wir an, Sie möchten sich entspannen. Meinen Sie, dass einer der } \\
\text { Klänge (oder beide) Sie dabei unterstützen könnte? }\end{array}$} \\
\hline ja: $70 \%$ & & & nein: $30 \%$ \\
\hline \multicolumn{4}{|c|}{ Welchen Klang würden Sie zum Entspannen lieber hören? } \\
\hline $1: 76 \%$ & 2: $14 \%$ & gleichgültig: $10 \%$ & \\
\hline
\end{tabular}

Tab. 4: Antworten bei Klangpaar 5

$44 \%$ der Teilnehmer, die mit „nein“ antworteten, begründeten dies mit der hohen Lage. Sie bevorzugten im vorangegangenen Klangpaar 4 (erster Klang gleich, zweiter 
Klang eine Oktave tiefer) den zweiten Klang wegen seiner Tiefe. Ebenfalls $44 \%$ der Hörer begründeten ihre Ablehnung mit der Bewegung, die sie als unruhig bzw. zu stark empfanden. Auch sie wählten - mit einer Ausnahme (Ablehnung beider Klänge) - im Klangpaar 4 den zweiten Klang wegen der tiefen Lage. In einem Fall waren die beiden Ablehnungsgründe verknüpft: „,zu hoch, schwingen zu viel““.

$81 \%$ der Hörer, die den ersten Klang bevorzugten, wählten ihn wegen der geringeren Bewegtheit. Dies ist der einzige oft genannte Grund. Bei jenen Teilnehmern, die den zweiten Klang vorzogen, tritt kein Entscheidungskriterium hervor.

Die Teilnehmer ohne Präferenz für einen der Klänge hörten keinen Unterschied. $52 \%$ aller Teilnehmer wiesen auf stärkere Bewegung des zweiten Klangs hin.

\subsection{Klangpaar 6: Klang_8, Klang_4}

In diesem Klangpaar ist der Unterschied der Bewegung besonders gut hörbar. Der erste Klang entspricht dem ersten Klang in Klangpaar 5. Er fluktuiert recht langsam, der zweite verklingt ohne Fluktuieren (Ergebnisse s. Tab. 5).

\begin{tabular}{|c|c|c|c|}
\hline $\begin{array}{l}\text { Nehmen } \\
\text { Klänge ( }\end{array}$ & $\begin{array}{l}\text { an, Sie } \mathrm{m} \\
\text { beide) } \mathrm{S}\end{array}$ & $\begin{array}{l}\text { n sich entspanne } \\
\text { ei unterstützen }\end{array}$ & ass einer der \\
\hline ja: $78 \%$ & & & nein: $22 \%$ \\
\hline Welcher & gg würde & zum Entspannen & \\
\hline $1: 85 \%$ & $2: 15 \%$ & gleichgültig: $0 \%$ & \\
\hline
\end{tabular}

Tab. 5: Antworten bei Klangpaar 6

$54 \%$ jener Hörer, die mit „nein“ antworteten, empfanden die Klänge als zu hoch. Dies ist der einzige häufig genannte Grund. Beim Klangpaar 4 (erster Klang gleich, zweiter Klang eine Oktave tiefer) wählten diese Teilnehmer - mit einer Ausnahme (Ablehnung beider Klänge) - den zweiten Klang wegen seiner Tiefe.

Von den Hörern, die bei der zweiten Frage den ersten Klang wählten, gaben $75 \%$ als Grund die Bewegung an; $20 \%$ begründeten ihre Auswahl damit, dass der zweite Klang intensiver, aufdringlich sei. Niemand verknüpfte beide Gründe. Wer den zweiten Klang lieber hören mochte, nannte (mit einer Ausnahme) die fehlende bzw. sehr geringe Bewegung als Grund. 


\subsection{Klangpaar 2: Klang_6, Klang_7}

Dieses Paar bietet verschieden hohe Klänge bei ähnlicher Bewegtheit. Der erste Klang ist eine Variante des ersten Klangs in den Paaren 1 und 3. Geänderte Schwebungsfrequenzen der ersten drei Teilschwingungen ergeben ein ruhigeres Bewegungsmuster. Der zweite Klang ist so gestaltet, dass er von einer kleinen, ähnlich angeschlagenen Schale stammen könnte, die eine Oktave höher klingt. Die Schwebungsfrequenzen bleiben gleich. Die simulierte kleine Schale kommt schneller zur Ruhe als die mittelgroße. Tab. 6 zeigt die Häufigkeitsverteilung der Antworten.

\begin{tabular}{|c|c|c|c|}
\hline \multicolumn{4}{|c|}{$\begin{array}{l}\text { Nehmen wir an, Sie möchten sich entspannen. Meinen Sie, dass einer de } \\
\text { Klänge (oder beide) Sie dabei unterstützen könnte? }\end{array}$} \\
\hline ja: $95 \%$ & & & nein: $5 \%$ \\
\hline \multicolumn{4}{|c|}{ Welchen Klang würden Sie zum Entspannen lieber hören? } \\
\hline $1: 63 \%$ & $2: 35 \%$ & gleichgültig: $2 \%$ & \\
\hline
\end{tabular}

Tab. 6: Antworten bei Klangpaar 2

Unter den Gründen für die Antwort „nein“ kommt nur die hohe Lage mehrfach vor: Den zweiten Klang beschrieben zwei Hörerinnen als „fast schrill“ bzw. „schrill“, ein Hörer nannte den ersten Klang „zu gläsern“. Niemand bezeichnete beide Klänge als zu hoch.

$53 \%$ der Teilnehmer, die den ersten Klang bevorzugten, nannten die tiefere Lage als Grund, $19 \%$ das längere Ausklingen und $14 \%$ die stärker empfundenen Schwebungen. In den meisten Fällen war einer dieser Gründe ausschlaggebend, nur wenige Hörer nannten zwei Merkmale. Von jenen Teilnehmern, die den zweiten Klang wählten, gaben $60 \%$ als Grund an, dieser Klang sei tiefer. Der zweite oft genannte Grund ist schwächeres Fluktuieren $(30 \%) ; 15 \%$ der Hörer, die sich für den zweiten Klang entschieden, erwähnten dies zusätzlich zum Merkmal „tiefer“, bei 15\% war das schwächere Fluktuieren allein ausschlaggebend.

Insgesamt gaben $20 \%$ der Beteiligten an, beim ersten Klang stärkeres Fluktuieren gehört zu haben.

\subsection{Klangpaar 4: Klang_8, Klang_9}

Im zweiten Paar mit verschieden hohen Klängen ist der erste Klang eine weitere Variante des ersten Klangs in den Paaren 1 und 3. Er ähnelt dem ersten Klang im 
zweiten Paar: Die Schwebungsfrequenzen der ersten drei Teilschwingungen sind gleich, die Schwebungsfrequenz der vierten Teilschwingung ist verringert. Der zweite Klang könnte von einer großen Schale stammen, die eine Oktave tiefer und ähnlich laut klingt. Dies erfordert einen etwas schwächeren Anschlag. Die Schwebungsfrequenzen bleiben gleich. Ein sechster Teilton ist kurz zu hören. Die simulierte große Schale klingt länger nach als die mittelgroße. Tab. 7 zeigt die Häufigkeitsverteilung der Antworten.

$\begin{aligned} & \text { Nehmen wir an, Sie möchten sich entspannen. Meinen Sie, dass einer der } \\
& \text { Klänge (oder beide) Sie dabei unterstützen könnte? } \\
& \text { ja: } 95 \%\end{aligned}$
\begin{tabular}{l|l|l|} 
Welchen Klang würden Sie zum Entspannen lieber hören? & \\
$\begin{array}{l}\text { 1: } 14 \% \\
\text { situationsabhängig: } 7 \%\end{array}$ & 2: $70 \%$ & \\
\hline
\end{tabular}

Tab. 7: Antworten bei Klangpaar 4

In den Kommentaren zur Antwort „nein“ kommt allein die Lage mehrfach vor: Der erste Klang erschien zu hoch, der zweite zu tief.

Teilnehmer, die den ersten Klang vorzogen, begründeten dies vor allem mit der höheren Lage (38\%). Dies ist das einzige mehrfach genannte Kriterium. Die Wahl des zweiten Klangs wurde nahezu ausschließlich mit der tieferen Lage begründet. Alle Hörer, die den Klang wählten, nannten diesen Grund; $10 \%$ von ihnen erwähnten zusätzlich ein schwächeres Pulsieren der Lautstärke. Das langsamere Verklingen kam nicht zur Sprache. Im Gegensatz zum zweiten Klangpaar gab es hier keine gegensätzlichen Aussagen zur Lage: Niemand bezeichnete den ersten Klang als tiefer.

\section{Diskussion}

\subsection{Alle Klangpaare, Frage 1}

Wie die Antworten auf die erste Frage zeigen, meinten die Versuchsteilnehmer in sehr vielen Fällen, dass einer der dargebotenen Klänge (oder beide) sie beim Entspannen unterstützen könnte. Offenbar wurde das Ziel erreicht, möglichst nur solche Personen einzubeziehen, die sich grundsätzlich mithilfe von Schalenklängen entspannen können.

In den Antworten zeichnen sich Grenzen für die Durchführung eines derartigen Versuchs ab. Offenbar beeinflusst die Reihenfolge der Klangpaare die Ergebnisse. Bei den Klangpaaren 1, 2 und 4 bejahten $93 \%$ bzw. $95 \%$ der Teilnehmer die erste Frage; 
bei den Klangpaaren 3, 5 und 6 liegt der Anteil zwischen $70 \%$ und $78 \%$. Im ersten und dritten Paar ist der erste Klang gleich, trotzdem lehnten beim dritten Paar sehr viel mehr Hörer als beim ersten Paar beide Klänge ab. Der einzige häufig genannte Grund ist die Klangbewegung. Nach den ruhiger bewegten Klängen des zweiten Paares erschien offenbar vielen Hörern die Bewegung auch des ersten Klangs im anschließenden Paar zu stark. Das vierte Klangpaar enthält den einzigen tiefen Klang des Versuchs. Eine große Mehrheit bevorzugte ihn wegen seiner Lage. Bei den anschließenden Paaren 5 und 6 lehnten $13 \%$ bzw. $12 \%$ der Hörer beide Klänge wegen der hohen Lage ab; bei den ersten drei Klangpaaren kam dies wesentlich seltener vor $(3 \%, 0 \%, 5 \%)$.

\subsection{Klangpaar 1}

Hier zeigt sich keine klare Präferenz für einen der Klänge. Die Anzahl der Antworten zugunsten des ersten und zugunsten des zweiten Klangs ist ähnlicher als bei allen anderen Klangpaaren. Mit „gleichgültig“ antworteten wesentlich mehr Personen als bei den anderen Klangpaaren; nur eine von ihnen hörte einen Klangunterschied, bezeichnete ihn aber als „marginal“ und sprach die Bewegungsgeschwindigkeit nicht an. Offensichtlich war der Klangunterschied für die meisten Beteiligten zu gering, um deutlich wahrgenommen zu werden.

\subsection{Klangpaar 3}

Die Entscheidung für einen der Klänge fällt hier eindeutig zugunsten des ersten aus, der Grund ist in fast $90 \%$ der Fälle die geringere Bewegung. Es gibt aber auch Hörer, die den zweiten Klang wegen seiner stärkeren Bewegtheit wählten.

Sehr viel mehr Beteiligte als bei den Klangpaaren 1 und 2 beantworteten die erste Frage mit „nein“. Zwei Drittel von ihnen empfanden die Bewegung beider Klänge als zu stark. Der zweite Klang ist zwar von allen bis zu dieser Stelle dargebotenen Klängen am stärksten bewegt, der erste gleicht aber dem ersten Klang in Klangpaar 1. Dass trotzdem so viele Hörer beide Klänge ablehnten, liegt wahrscheinlich am zuvor gehörten Paar 2, dessen Klänge langsamer bewegt sind. Man kann hierin einen zusätzlichen Hinweis darauf sehen, dass sanft bewegte Klänge eher zum Entspannen ausgewählt werden als stärker bewegte. 


\subsection{Klangpaar 5}

Mehr Hörer als bei allen anderen Klangpaaren verneinten die erste Frage. $44 \%$ dieser Teilnehmer begründeten ihre Ablehnung mit der hohen Lage; sie wählten im vorangegangenen Klangpaar den zweiten Klang wegen seiner Tiefe, insofern ist die Ablehnung konsequent. Ebenso viele Personen verneinten die erste Frage wegen der Bewegtheit beider Klänge. Dies mag auf den ersten Blick überraschen, weil die Bewegung des ersten Klangs sich kaum von der Bewegung der Klänge im vorangegangenen Paar unterscheidet (die ersten Klänge sind in beiden Paaren gleich). Bei näherem Hinsehen erscheinen die Antworten jedoch plausibel: Beim Klangpaar 4 wählten diese Hörer mit einer Ausnahme (Ablehnung beider Klänge) den zweiten Klang wegen seiner tiefen Lage. Dieses Kriterium stand offenbar so im Vordergrund, dass die Klangbewegung keinen Anlass zur Ablehnung bot. Insgesamt wiesen beim fünften Klangpaar $52 \%$ der Teilnehmer auf stärkere Bewegung des zweiten Klangs hin weniger als beim dritten Klangpaar (68\%), obwohl beim fünften zusätzlich zur Verdopplung der Schwebungsfrequenz von Teilschwingung 2 auch die Schwebungsfrequenz der dritten Teilschwingung verdoppelt ist. Den Unterschied der Bewegtheit sprachen wahrscheinlich deswegen weniger Hörer an, weil die hohe Lage ein ebenso wichtiger Ablehnungsgrund war wie die Bewegung, während beim dritten Klangpaar die Bewegung sehr viel häufiger genannt wurde als die Lage.

Unter jenen Beteiligten, die auf die erste Frage mit ,ja“ antworteten, sind die Präferenzen ähnlich verteilt wie beim dritten Klangpaar. Für den ersten Klang entschieden sich gut fünfmal mehr Personen als für den zweiten, vier Fünftel von ihnen nannten die geringere Bewegtheit als Grund.

\subsection{Klangpaar 6}

Mehr als ein Fünftel der Hörer antwortete auf die erste Frage mit „nein“. Als Grund nannten diese Teilnehmer vor allem die hohe Lage. Beim Klangpaar 4 wählten sie - mit einer Ausnahme (Ablehnung beider Klänge) - den zweiten Klang wegen seiner Tiefe. Ihre Vorliebe für den tiefen Klang wirkte wohl noch beim sechsten Klangpaar nach.

Die Präferenz für einen der Klänge als Entspannungshilfe ist hier unter allen Klangpaaren am größten. Drei Viertel jener Hörer, die den ersten Klang vorzogen, begründeten dies mit der Bewegung. Weitere $20 \%$ nannten als Grund die Intensität bzw. Aufdringlichkeit des zweiten Klangs. Es liegt nahe, das vergleichsweise starre 
Ausklingen als Ursache anzusehen. Andererseits bevorzugte eine Minderheit gerade wegen der fehlenden Fluktuation den zweiten Klang.

\subsection{Klangpaar 2}

Fast alle Teilnehmer beantworteten die erste Frage mit ,ja“. Der erste Klang wurde in erster Linie wegen der tieferen Lage bevorzugt, das längere Ausklingen tritt demgegenüber in den Hintergrund. Jene Teilnehmer, die den zweiten Klang vorzogen, orientierten sich ebenfalls in erster Linie an der Lage. Sie nahmen den zweiten Klang tiefer wahr. Die Klangeigenschaft „tiefer“ als Entscheidungskriterium steht somit in beiden Gruppen an erster Stelle. Bemerkenswert ist, dass viele Hörer den zweiten Klang als tiefer empfanden. Hierfür sind verschiedene Ursachen denkbar. Der Grundton ist beim ersten Klang tiefer, gleichzeitig aber sind die hohen Teiltöne (4 und 5) stärker als der höchste Teilton (3) des zweiten Klangs (der etwa um einen Ganzton unter dem höchsten Teilton des ersten Klangs liegt). Je nachdem, welche Eigenschaft man vorwiegend wahrnimmt, kann man den ersten Klang als tiefer oder höher (heller) empfinden. Beim ersten Klang treten die Teiltöne 2 und 3 hervor, beim zweiten die Teiltöne 1 und 2. Die zweitgenannten Töne sind ähnlich hoch - Teilton 3 bei Klang 1 liegt um einen Halbton tiefer als Teilton 2 bei Klang 2 -, aber Teilton 2 bei Klang 1 liegt um einen Tritonus höher als Teilton 1 bei Klang 2. Wenn diese Wahrnehmung im Vordergrund steht, erscheint Klang 2 tiefer als Klang 1.

Der nennenswerte Anteil von Teilnehmern, die sich für den zweiten Klang entschieden, lässt erkennen, dass auch eine kleine Klangschale zum Entspannen in Frage kommt. Um als hinreichend „tief“ gehört zu werden, sollte sie dickwandig sein der Klang ist dann dunkler - und mit einem gepolsterten Schlägel maßvoll angeschlagen werden. Besser geeignet erscheint im direkten Vergleich allerdings die mittelgroße Schale, die um $80 \%$ mehr Hörer bevorzugten als die kleine. Das längere Ausklingen trägt hierzu ein wenig bei.

Ein Fünftel der Hörer sagte, das Fluktuieren sei beim ersten Klang stärker. Dies liegt vermutlich an den nur hier vorhandenen Teilschwingungen 4 und 5, die mit Schwebungsfrequenzen von 5,7 bzw. $8,9 \mathrm{~Hz}$ rascher pulsieren als die anderen Teilschwingungen. 


\subsection{Klangpaar 4}

Nahezu alle Teilnehmer bejahten die erste Frage. Die größere Schale wählten fünfmal mehr Personen als die kleinere, alle nannten den tieferen Klang als Grund; ein kleiner Anteil erwähnte außerdem ein schwächeres Fluktuieren. Das langsamere Verklingen der größeren Schale hatte keine Bedeutung. Diese Ergebnisse zeigen eine klare Vorliebe für den tieferen Klang. Dabei sollte man aber nicht übersehen, dass einige Hörer den ersten Klang gerade wegen seiner höheren Lage wählten und manche Teilnehmer je nach Situation entscheiden würden.

\subsection{Fazit}

Um den Klang einer Klangschale als Entspannungshilfe zu nutzen, ist es für eine deutliche Mehrheit der Versuchsteilnehmer wichtig, dass der Klang fluktuiert. Die Fluktuation sollte vorzugsweise recht langsam verlaufen. Doch gibt es Minderheiten unter den Hörern, die gleichförmig verklingende bzw. rascher fluktuierende Klänge zum Entspannen vorziehen. Auch beim zweiten Klangpaar - dem Vergleich von Schalen gewidmet, die in verschiedenen Tonlagen unterschiedlich lang ausklingen gaben manche Hörer an, sich wegen stärkeren bzw. schwächeren Fluktuierens für einen der Klänge entschieden zu haben. Beim Vergleich verschieden großer Schalen ist die Präferenz ebenfalls nicht einheitlich. Eine deutliche Mehrheit würde zum Entspannen lieber eine größere Schale hören. Ausschlaggebend ist der tiefere Klang, das längere Ausklingen tritt in den Hintergrund. Andererseits möchten sich manche Personen lieber mit einem höheren Klang entspannen oder je nach Situation entscheiden. Man kann keine ,idealen“ Klangeigenschaften bestimmen für eine Schale, deren Klänge als Entspannungshilfe dienen sollen.

Diese aus dem Hörversuch abgeleiteten Erkenntnisse haben Konsequenzen für die Kriterien, die man beim Kauf einer Klangschale beachten sollte. Ein wichtiges Merkmal ist die Tonlage. Sie hängt von der Schalengröße und von der Wandstärke ab. Bei gleicher Tonlage begünstigt ein dicker Rand einen dunkel gefärbten Klang, weil die Obertöne schwächer ausgeprägt sind. Dies erreicht man außerdem mit einem weichen Schlägel und mit sanftem Anschlag. Besonderes Augenmerk verdient die Geschwindigkeit der Klangfluktuation. Sie kann von Schale zu Schale sehr verschieden sein (Thies 2011). Das Fluktuieren ist je nach Anschlagstelle stärker oder schwächer ausgeprägt. Es ist vorteilhaft, sich nacheinander auf die einzelnen Teiltöne einer Schale 
zu konzentrieren und jeweils viele verschiedene Anschlagstellen zu erproben - auch mit hartem Schlägel, um die hohen Teiltöne kräftiger hören zu können.

\section{Danksagung}

Allen, die am Versuch teilgenommen haben, danke ich für ihre Mitwirkung.

\section{Literatur}

(Weitere Literatur ist verzeichnet in Thies 2010. Neuauflagen und Übersetzungen bereits früher genannter Bücher sind hier nicht aufgeführt.)

Aarts, Ronald M.; Ouweltjes, Okke; Bulut, Murtaza (2014): An electro-acoustic implementation of Tibetan bowls: Acoustics and perception. In: Noise \& Vibration Worldwide 45, No. 1, S. 12-23; http://nvw.sagepub.com/content/45/1/ 12.full.pdf, eingesehen am 14.10.2016.

Bidin, Livia; Pigaiani, Luca; Casini, Manlio; Seghini, Pietro; Cavanna, Luigi (2016): Feasibility of a trial with Tibetan Singing Bowls, and suggested benefits in metastatic cancer patients. A pilot study in an Italian Oncology Unit. In: European Journal of Integrative Medicine 8, S. 747-755; http://dx.doi.org/ 10.1016/j.eujim.2016.06.003 [als „Corrected Proof“ online veröffentlicht am 15.06.2016, eingesehen am 13.10.2016].

Braun, M.; Braun, A. (2015): Klangschalen-Entspannung bei Fibromyalgie im Rahmen einer multimodalen Schmerztherapie: Eine quasi randomisierte Studie. In: Die Medizinische Welt 66, S. 195-199.

Collin, Samantha R.; Keefer, Chloe L.; Moore, Thomas R. [2015]: The etiology of chatter in the Himalayan singing bowl. In: Proceedings of the 3rd Vienna Talk on Music Acoustics, „Bridging the gaps“, September 16-19 2015. Hrsg. v. Alexander Mayer, Vasileios Chatziioannou u. Werner Goebl. Wien: Universität für Musik und darstellende Kunst, S. 120-123; http://viennatalk2015.mdw.ac.at/ ?page $\mathrm{id}=13002 \& \mathrm{pap}=64$, eingesehen am 27.09.2016. 
Draxler-Zenz, Tanja (2016): Achtsame Klangschalen-Spiele: Spielerisch Ruhe und Entspannungsfähigkeit in Kita und Grundschule fördern. Münster: Ökotopia.

Dülmen, Beate van (2013): Klingen, spüren, schwingen: Fantasiereisen mit der Klangschale: kindgerechte Spiele, Körperübungen, Klangmassagen, Rituale und Lieder zur Stärkung von Selbstbewusstsein und Selbstvertrauen in Kiga und Grundschule. Münster: Ökotopia.

Hess, Emily (2016): KlangYoga: Freude - Entspannung - Rhythmus: Der Weg zur inneren Freiheit. Oberstdorf: Windpferd.

Hess, Peter [2015]: Klangschalen: Mein praktischer Begleiter. Schüttorf: Hess.

Lahner, Regina (2012): Klangschalenmassage leicht gemacht: Theorie und Praxis; Schritt für Schritt Anleitung; Einfach - Klar - Verständlich. Norderstedt: Books on Demand.

Lahner, Regina (2013): Meditationen mit Klangschalen leicht gemacht. Norderstedt: Books on Demand.

Lahner, Regina (2015): Neue Meditationen mit Klangschalen leicht gemacht. Norderstedt: Books on Demand.

Landry, Jayan Marie (2015): Healing emotional trauma: Practical pathways to decrease anxiety, anger \& lower blood pressure. Bloomington, IN: Balboa Press.

Landry, Jayan M[arie] (2012): Measuring the effects of a Himalayan singing bowl on a meditation practice: A quantitative approach. $\mathrm{PhD}$ dissertation, Capella University.

Landry, Jayan Marie (2014): Physiological and psychological effects of a Himalayan singing bowl in meditation practice: A quantitative analysis. In: American Journal of Health Promotion 28, S. 306-309.

Leon, Emile de (2012): The mastery book of Himalayan singing bowls: A musical, spiritual and healing perspective. Middletown, CT: Temple Sounds. 
Lindner, David [2014]: Der Klang der Liebe: Ein Jahrtausendritual. Klangschalen rund um Zeugung, Schwangerschaft, Geburt und das erste Lebensjahr sowie eine Klangübung, die die Welt verändern wird. Battweiler: Traumzeit.

Lindner, David; Mempel, Uta Karen (2008): Klangschalen für Wellness und Sauna: Klangübungen und Klangzeremonien in Saunen, Bädern und Ruheräumen. Für daheim, zur Begleitung von Gruppen, für den professionellen Einsatz durch Saunameister. Traumklänge - Klangreisen mit Klangschalen. Zur Entspannung, Harmonisierung und Vitalisierung. [Battweiler]: Traumzeit.

Mayer, Ilona Varta (2013): Heilsame Schwingungen: Klangschalenmassage für einen gesunden Rücken und mehr Wohlbefinden. Darmstadt: Schirner.

Mempel, Uta Karen (2012): Die Welt der Klänge: Klangschalen und -instrumente für Klangreisen. München: Grin.

Meyer, Andrzej; Portalski, Marek (2013): The method of multitone parameters determining selected idiophones. In: 2013 Signal Processing: Algorithms, Architectures, Arrangements, and Applications (SPA 2013). Poznan, Poland, 26-28 September. Piscataway, NJ: IEEE, S. 191-194.

Oberle, Horst (2013): Die Kraft der Klangschalen. Güllesheim: Silberschnur.

Pawłowski, Paweł; Portalska, Halina; Portalski, Marek (2013): Nonharmonic multitone of singing bowls and digital signal generators. In: 2013 Signal Processing: Algorithms, Architectures, Arrangements, and Applications (SPA 2013). Poznan, Poland, 26-28 September. Piscataway, NJ: IEEE, S. 186-190.

Perry, Frank (2014): Himalayan sound revelations: The complete singing bowl book. London: Polair.

Pöttinger, Helga (2014): Harmonie und Heilkraft mit Alchemie Kristallklangschalen. Norderstedt: Books on Demand. 
Smutný, Jaroslav; Pazdera, Luboš (2014): Acoustic vibration analysis of a Tibetan singing bowl - Akusticko vibrační analýza tibetské mísy. In: Akustika 22, S. $37-43$.

Stamou, Vasileios; Chatzoudi, Theano; Stamou, Lelouda; Romo, Lucia; Graziani, Pierluigi (2016): Music-assisted systematic desensitization for the reduction of craving in response to drug-conditioned cues: A pilot study. In: The Arts in Psychotherapy 51, S. 36-45.

Terwagne, Denis; Bush, John W. M. (2011): Tibetan singing bowls. In: Nonlinearity 24, S. R51-R66.

Thies, Wolfgang (2008): Reaktionen auf das Hören einer Klangschale. In: Musik-, Tanz- und Kunsttherapie 19, S. 83-92.

Thies, Wolfgang (2010): Stereofone Synthese von Klangschalenschwingungen. In: Frankfurter Zeitschrift für Musikwissenschaft 13, S. 38-54; http://www.europeanmusicology.eu/2010-13-jahrgang, eingesehen am 14.10.2016.

Thies, Wolfgang (2011): Variabilität von Klangschalenschwingungen: Beobachtungen mithilfe von Analyse und Synthese. In: Frankfurter Zeitschrift für Musikwissenschaft 14, S. 26-45; http://www.european-musicology.eu/2011-14jahrgang, eingesehen am 14.10.2016.

Wulf, Adalgis [David Lindner] [2014]: Die Kunst der Klangmassage: Das Klangmassage-Praxisbuch für Klangmasseure, Therapeuten, Heiler und Musiker. [Klangmassage mit Klangschalen Schritt für Schritt erlernen und professionell umsetzen.] Battweiler: Traumzeit.

Wulf, Adalgis [David Lindner] [2016]: Praxisbuch Klangschale: Lass es dir gutgehn. Battweiler: Traumzeit. 